\section{Postdiscrimination stimulus generalization in humans: An extension of Galizio and Baron}

\author{
K. GEOFFREY WHITE and DAVID R. THOMAS \\ University of Colorado \\ Boulder, Colorado 80309
}

A characteristic consequence of intradimensional discrimination training is a peak shift in the gradient of stimulus generalization (Purtle, 1973). Maximal responding occurs to a stimulus value removed from $\mathrm{S}+$ in the direction away from $\mathrm{S}-$. Peak shift is commonly interpreted as evidence for the summation of excitatory and inhibitory generalization gradients (cf. Spence, 1937). Thomas (1974) has accounted for peak shift (in human generalization) in terms of a change in adaptation level (AL) from training to test. This interpretation was originally developed to explain the "central tendency effect", i.e., a peak shift toward the center of an asymmetrical test series following single stimulus training (cf. Helson \& Avant, 1967, Thomas \& Jones, 1962, etc.). In the case of two-stimulus discrimination training, $\mathrm{AL}$ is presumably established midway between the training stimuli, $\mathrm{S}+$ and $\mathrm{S}-$, and the subject learns to respond to a stimulus $(\mathrm{S}+)$ which is, say, $\mathrm{X}$ units greater than the AL. In generalization testing, AL moves towards the central value in the test series, which is normally the previous $\mathrm{S}+$. Thus, the stimulus value associated with maximal responding during testing is $\mathrm{X}$ units greater than the new (test) $\mathrm{AL}$, thereby resulting in the peak shift.

Galizio and Baron (1979) recently reported a study in which human subjects were trained to discriminate between two tones (both $\mathrm{S}+$ ) and a third tone (S-) located midway between the $\mathrm{S}+$ tones. Tones presented in a generalization test were symmetrical around $\mathrm{S}-$, extending beyond the $\mathrm{S}+$ values. Thus no change was expected in AL from training to test, and therefore no peak shift should have occurred. The gradients obtained were $\mathrm{V}$-shaped, with maximal responding to tones maximally displaced from the S+ values. Galizio and Baron (1979) concluded that their generalization data exhibited peak shift, contrary to the AL hypothesis but consistent with the excitation-inhibition formulation of Spence (1937). Their conclusion is debatable, however, in that peak shift is normally characterized by a distinct mode

This research was supported by NSF Grant BNS 78-01407 to David R. Thomas. K. Geoffrey White was on sabbatical leave from Victoria University, Wellington, New Zealand. Robert J. Newlin assisted in the collection of data. Requests for reprints should be addressed to: Dr. David R. Thomas, Department of Psychology, University of Colorado, Bouider, Colorado 80309. at a value displaced from $\mathrm{S}+$, along with lower response rates to stimuli more extreme than the modal values. The $V$-shaped gradients could have resulted from the use of a relational strategy, with subjects learning to respond to all values higher or lower than the central S-. Adoption of such a strategy would be inconsistent with both the AL and the excitation-inhibition positions. To eliminate this possibility, we replicated Galizio and Baron's experimental condition with a stimulus dimension (brightness) for which empirical AL (or central tendency) effects have clearly been demonstrated (Newlin, Rodgers, Dickson, Strub, \& Thomas, 1978; Thomas, Strub, \& Dickson, 1974) and with explicit instructions to respond only to the two $\mathrm{S}+$ values. Six male and six female undergraduate students served. Apparatus and stimuli were the same as those used by Newlin et al., 1978. A white light could be presented at one of 11 different intensities (logarithmically) equally spaced. Stimulus 1 (S1) was the dimmest of the test values, S11 the brightest. Instructions indicated that subjects would be required to remember the first two brightnesses they were shown, because they would have to identify them iater. They were told to say "same" during subsequent presentations of those two stimuli and "different" to all others. They were also told that they would receive feedback only on early trials.

Throughout training and testing, 5-sec stimulus presentations were separated by intertrial intervals averaging $3 \mathrm{sec}$. Following initial presentation of stimuli S4 and S8, 42 discrimination trials comprising 14 blocks of three stimuli, S4, S6, and S8, were conducted. In discrimination training, the subject's response "same" to S4 and S8 or "different" to S6 led to the experimenter's feedback, "correct." Feedback for incorrect responses was "No, that was different from both of the test brightnesses" or "No, that was the same as one of the test brightnesses". A generalization test immediately followed discrimination. The test was composed of four randomized blocks of the 11 stimulus values. There was no feedback during the test.

Figure 1 presents the frequencies of "same" responses to each test stimulus value summed over subjects. The gradient exhibits a minimum at the S- value, S6, and maxima at values $S 3$ and S9, with responding dropping off sharply to the more peripheral stimuli. Every subject responded more to S9 than to S11, and all but one responded more to S3 than to S1 (ps $<.01$, binomial test). Each subject's gradient was analyzed for the location of two modes, one on each side of $\mathrm{S}-$. Ties in number of "same" responses were credited as $1 / 2$ mode to each of two stimuli or $1 / 3$ to each of three. Looking first 


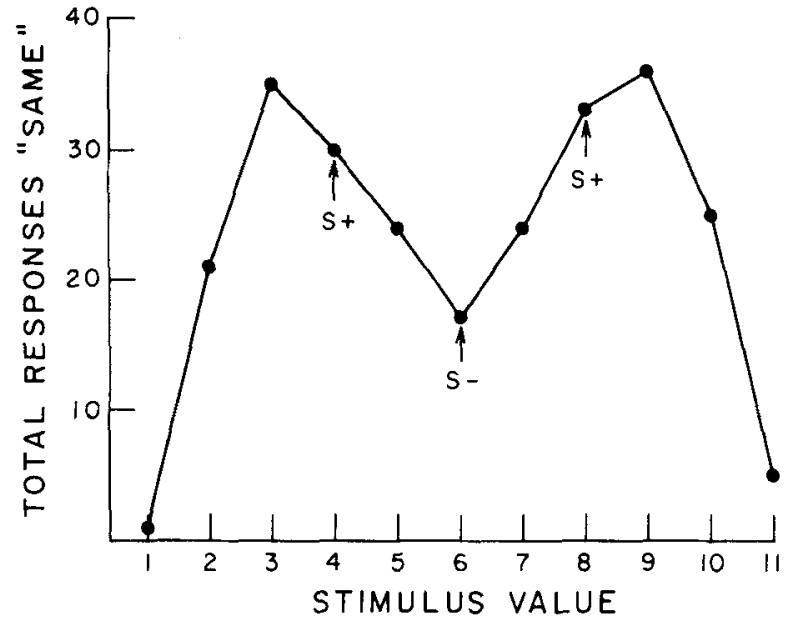

Figure 1. Generalization of "same" responses after three-stimulus $(\mathbf{S}+\mathbf{S}-\mathbf{S}+$ ) discrimination training.

at the left wing of the gradient, 5 modes fell at S3, the peak-shifted stimulus, and $2 \frac{1 / 2}{2}$ modes fell at $S 2$, the stimulus still farther removed from $S-$. Less than 2 modes fell at any other value, including that of S4, the $\mathrm{S}+$. The picture was similar for the right wing of the gradient. Six modes fell at S9, the peak-shifted stimulus value. Only $3 \frac{1 / 2}{2}$ modes fell at $\mathrm{S} 8$, the $\mathrm{S}+$, with fewer than 2 modes falling anywhere else. This record of individual performance is very consistent with the group average gradient shown in Figure 1. Overall, these data provide convincing evidence of bidirectional peak shift for human generalization along the dimension of brightness.

Galizio and Baron (1979) implied that Spence's (1937) excitation-inhibition theory, which predicts the bidirectional peak shift, is therefore a better account of stimulus generalization of a voluntary response in humans. It certainly is a better account of the bidirectional peak shift. Recall, however, that AL theory was originally developed to explain the central tendency effect, which has been replicated many times with many different stimulus dimensions (cf. Thomas, 1974). This phenomenon is, of course, inconsistent with Spence's theory and it demonstrates that no view of human generalization can be complete which fails to take into account the constitution of the generalization test series.

Clearly, neither the excitation-inhibition view nor the AL view provides a satisfactory account of generalization in humans. Perhaps both learning and perceptual mechanisms are involved, depending on the paradigm or even the parameters employed. To illustrate this final point, Newlin, Rodgers, and Thomas (1979) have reported a study in which AL theory predicted a peak shift in one direction, whereas Spence's theory predicted one in the other. The results were consistent with an $\mathrm{AL}$ interpretation when minimal training was given, but indicated the presence of both AL and Spence-type processes when training was extensive.

\section{REFERENCES}

Gal1z10, M., \& Baron, A. Human postdiscrimination gradients: The effects of three-stimulus discrimination training. Animal Learning \& Behavior, 1979, 7, 53-56.

Helson, H., \& Avant, L. J. Stimulus generalization as a function of contextual stimuli. Journal of Experimental Psychology, $1967,73,565-567$.

Newlin, R. J., Rodgers, J. P., Dickson, J. F., Jr., Strub, H., \& Thomas, D. R. The central tendency effect in stimulus generalization: Effects of establishing a "preexperimental" frame of reference. Perception \& Psychophysics, 1978, 24, 161-167.

Newlin, R. J., Rodgers, J. P., \& Thomas, D. R. Two determinants of the peak shift in human voluntary stimulus generalization. Perception \& Psychophysics, 1979, 25, 478-486.

Purtle, R. B. Peak shift: A review. Psychological Bulletin, $1973,80,408-421$

Spence, K. W. The differential response in animals to stimuli varying within a single dimension. Psychological Review, 1937, 44, 430-444.

Thомаs, D. R. The role of adaptation-level in stimulus generalization. In G. Bower (Ed.), The psychology of learning and motivation (Vol. 8). New York: Academic Press, 1974.

Thomas, D. R., \& Jones, C. G. Stimulus generalization as a function of the frame of reference. Journal of Experimental Psychology, 1962, 64, 77-80.

Thomas, D. R., Strub, H., \& Dickson, J. F., Jr. Adaptationlevel and the central tendency effect in stimulus generalization. Journal of Experimental Psychology, 1974, 103, 446-474.

(Received for publication January 9, 1979; revision accepted April 11, 1979.) 